\title{
BOUNDED BAER-LEVI SEMIGROUPS
}

\author{
DIANA LINDSEY and BERNARD MADISON
}

(Received 13 November 1975)

Communicated by T. E. Hall

\begin{abstract}
Let $p$ and $q$ be infinite cardinal numbers, $p \geqq q, X$ a set of cardinality $p$, and $B L(X, p, q)$ the Baer-Levi semigroup of type $(p, q)$ on $X$. Subsemigroups of $B L(X, p, q)$ are defined and called bounded Baer-Levi semigroups. These semigroups are right simple and are universal in the embedding sense for the class of idempotent-free, right cancellative semigroups $S$ so that any two elements of $S$ have a common right identity in $S$. Further properties of bounded Baer-Levi semigroups are given and the structure of their lattices of congruences is discussed.
\end{abstract}

Any right cancellative idempotent-free semigroup can be embedded in a Baer-Levi semigroup (Baer and Levi (1932) or Clifford and Preston (1967)). Here we investigate what we call bounded Baer-Levi semigroups which turn out to be universal in the embedding sense for the class of right cancellative idempotent-free semigroups in which any two elements have a common right identity. This class of semigroups arises when considering certain semigroup actions (Lindsey (1974) and (1975)).

In Section 1 the definitions and basic properties of a bounded Baer-Levi semigroup are given. Sections 2 and 3 contain a discussion of the lattice of congruences on a bounded Baer-Levi semigroup. Aside from using some lemmas from Lindsey and Madison (1976) and standard results and terminology from Clifford and Preston (1961) and (1967) this discussion is selfcontained. The authors are grateful to A. H. Clifford who read the original version of this paper and made helpful suggestions for improvements.

Portions of this paper are from the first author's Ph.D. dissertation. The second author was partially supported by the National Science Foundation. 


\section{Bounded Baer-Levi semigroups}

Let $\boldsymbol{N}_{0}$ be the cardinal number of a countably infinite set, and let $p$ and $q$ be cardinal numbers with $p \geqq q \geqq \kappa_{0}$.

Let $X$ be a set with cardinal number $p$, i.e., $|X|=p$. The Baer-Levi semigroup of type $(p, q)$ on $X$, noted here $B L(X, p, q)$, is the subsemigroup of the full transformation semigroup on $X$ consisting of one-to-one functions $a: X \rightarrow X$ so that $|X \backslash X a|=q$. It is known, Chapter 8 of Clifford and Preston (1967), that $B L(X, p, q)$ is a right cancellative, right simple semigroup without idempotents. In fact, any right cancellative, idempotent-free semigroup $S$ is a subsemigroup of the Baer-Levi semigroup of type $(|S|,|S|)$. Information about the lattice of congruences on a Baer-Levi semigroup has been given by Šutov (1961) and Mielke, (1972), (1973) and (1975). A complete description of this lattice is given by Lindsey and Madison (1976).

Here we are interested in certain subsemigroups of $B L(X, p, q)$. Let be a nonempty set of subsets of $X$ satisfying

(B1) For each $F \in \mathscr{F},|F|=p$ and $|X| F \mid=q$;

(B2) For each pair $F_{1}, F_{2} \in \mathscr{F}$ there is $F \in \mathscr{F}$ so that $F_{1} \cup F_{2} \subset F$ and $\left|F \backslash F_{1} \cup F_{2}\right|=q$.

Such a collection will be called a bounding collection for $X$. We define the bounded Baer-Levi semigroup of type $(p, q)$ on $X$ with respect to $\mathscr{F}$, noted $B B L(X, p, q, \mathscr{F})$, to be the subsemigroup of $B L(X, p, q)$ consisting of functions $a: X \rightarrow X$ so that there is $F \in \mathscr{F}$ with $X a \subset F$.

Proposition 1.1. Let $B=B B L(X, p, q, \mathscr{F})$ be a bounded Baer-Levi semigroup. Then $B$ is (a) right cancellative, (b) idempotent-free, and (c) right simple, and (d) any two elements of $B$ have a common right identity.

Proof. Statements (a) and (b) follow because $S$ is a subsemigroup of $B L(X, p, q)$, and (c) and (d) are routine consequences of conditions (B1) and (B2) on $F$.

Proposition 1.2. If $S$ is an idempotent-free, right cancellative semigroup in which any two elements have a common right identity then $S$ is isomorphic to a subsemigroup of a bounded Baer-Levi semigroup of type $(|S|,|S|)$.

Proof. It is known, Clifford and Preston (1967), that there is a set $X$, $|X|=|S|$, with $S$ embedded in $B L(X,|S|,|S|)$. Consider $S$ as a subsemigroup of $B L(X,|S|,|S|)$ and let $\mathscr{F}=\{X a: a \in S\}$. Now for each $F \in \mathscr{F}$, $|F|=|X|$, and if $F_{1}, F_{2} \in \mathscr{F}$ let $X a=F_{1}$ and $X b=F_{2}$. If $c$ is a common right identity for $a$ and $b$ then $c$ is the identity function when restricted to $F_{1} \cup F_{2}$. Since $X c=F \in \mathscr{F}$ we must have $|F| F_{1} \cup F_{2}|=| X \mid$. Consequently $F$ is a bounding collection. Now by design $S \subset B B L(X,|S|,|S|, F)$. 
We note that there is not necessarily a unique bounding collection that determines a bounded Baer-Levi semigroup. Let $\mathscr{F}$ and $\mathscr{G}$ be two bounding collections for $X$. Clearly if $\mathscr{F} \subset \mathscr{G}$ then $B B L(X, p, q, \mathscr{F}) \subset B B L(X, p, q, \mathscr{G})$; in fact, if for $F \in \mathscr{F}$ there is $G \in \mathscr{G}$ so that $F \subset G$ then $B B L(X, p, q, \mathscr{F}) \subset$ $B B L(X, p, q, \mathscr{G})$. If, in addition, for each $G \in \mathscr{G}$ there is $F \in \mathscr{F}$ so that $G \subset F$ then $B B L(X, p, q, \mathscr{F})=B B L(X, p, q, \mathscr{G})$. Consequently any two such "interlaced" bounding collections determine the same bounded Baer-Levi semigroup. Conversely, any two bounding collections $\mathscr{F}$ and $\mathscr{G}$ so that $B B L(X, p, q, \mathscr{G})=B B L(X, p, q, \mathscr{F})$ are interlaced. Among all bounding collections that are interlaced with a given $\mathscr{F}$ there is a unique maximal one, $\bar{F}$. It is easy to see that $\overline{\mathscr{F}}$ is the set of ranges of elements of $B B L(X, \dot{p}, q, \mathscr{F})$. On the other hand, there is not always a bounding collection that is minimal with respect to being interlaced with $\mathscr{F}$, but there is one of smallest cardinality whose cardinal number we will designate by $c(\mathscr{F})$.

A one-to-one function $\Phi$ from $X$ onto $X$, i.e., a permutation of $X$, induces an isomorphism $\hat{\Phi}: B L(X, p, q) \rightarrow B L(X, p, q)$ given by $a \hat{\Phi}=\Phi^{-1} a \Phi$. Now if $\mathscr{F}$ is a bounding collection for $X$ then $\mathscr{F} \Phi=\{F \Phi: F \in \mathscr{F}\}$ is a bounding collection for $X$ and $B B L(X, p, q, \mathscr{F}) \hat{\Phi}=B B L(X, p, q, \mathscr{F} \Phi)$, i.e., the conjugate by permutations of bounded Baer-Levi semigroups are bounded Baer-Levi semigroups. Whether or not every pair of isomorphic bounded Baer-Levi subsemigroups of $B L(X, p, q)$ are conjugates is unknown to the authors. A related problem is whether or not every automorphism of $B L(X, p, q)$ is inner, i.e., induced by a permutation of $X$. We give below two non-isomorphic bounded Baer-Levi subsemigroups of $B L\left(X, \boldsymbol{\aleph}_{0}, \boldsymbol{\aleph}_{0}\right)$ where $X$ is a countable set. We drop the $\boldsymbol{N}_{0}$ 's to simplify notation.

EXAMPLE 1.3. Let $X$ be the set $\{(m, n): m$ and $n$ are positive integers $\}$. Let $\mathscr{F}$ be any countable tower that satisfies the conditions (B1) and (B2) of a bounding collection, say $\mathscr{F}=\left\{F_{1}, F_{2}, \cdots\right\}$ where $F_{i} \subset F_{i+1}$ for each $i$. Now there is a sequence, $e_{1}, e_{2}, \cdots$, of elements of $B B L(X, \mathscr{F})$ so that $e_{i}$ is the identity when restricted to $F_{i}$. Consequently if $a \in B B L(X, \mathscr{F})$ there is an $n$ so that $a e_{n}=a$. Clearly any isomorphic copy of $B B L(X, \mathscr{F})$ contains such a sequence. Conversely, if $B B L(X, \mathscr{G})$ is a bounded subsemigroup of $B L(X)$ and there is such a sequence, say $f_{1}, f_{2}, \cdots$, let $\mathscr{G}^{\prime}=\left\{G_{n} \in \mathscr{G}: X f_{n} \subset G_{n}\right.$ for $n=$ $1,2,3, \cdots\}$. It is easy to see that $\mathscr{G}^{\prime}$ is a bounding collection for $X$, and since $\mathscr{G}^{\prime} \subset \mathscr{G}, B B L\left(X, \mathscr{G}^{\prime}\right) \subset B B L(X, \mathscr{G})$. If $a \in B B L(X, \mathscr{G})$ then there is $n$ so that $a f_{n}=a$ and consequently $X a \subset G_{n} \in \mathscr{G}^{\prime}$. Thus $B B L\left(X, \mathscr{G}^{\prime}\right)=B B L(X, \mathscr{G})$. In particular we have seen that $\mathscr{G}$ contains a subset $\mathscr{G}^{\prime}$ which is a tower, and if $G \in \mathscr{G}$, there is $G^{\prime} \in \mathscr{G}^{\prime}$ so that $G \subset G^{\prime}$. Now let $\mathscr{H}$ be the collection of subsets of $X$ that are finite unions of graphs of functions from the positive inte- 
gers to the positive integers. Clearly $\mathscr{H}$ is a bounding collection that does not contain a tower as above. Consequently $B B L(X, \mathscr{F})$ and $B B L(X, \mathscr{H})$ are not isomorphic.

\section{Difference set congruences on bounded Baer-Levi semigroups}

In this and the next section the lattice of congruences on bounded Baer-Levi semigroups is discussed. If $X$ is an infinite set we will say that a bounding collection $\mathscr{F}$ for $X$ is an effective bounding collection if $\cup \mathscr{F}=X$. Except in the following result, which justifies the term "effective", we will assume that any bounding collection is effective.

Proposition 2.1. Let $B=B B L(X, p, q, \mathscr{F})$ with $\cup \mathscr{F}$ not necessarily all of $X$. Let $\rho=\{(a, b) \in B \times B: s a=s b$ for each $s \in B\}$. Then $\rho$ is a congruence on $B$ and $B / \rho$ is isomorphic to $B B L(\cup \mathscr{F}, p, q, \mathscr{F})$.

Proof. It is well known and routine to verify that $\rho$ is a congruence. For $a \in b$ let $a \rho$ denote the $\rho$-class of $a$. Define $\Phi: B / \rho \rightarrow B B L(\cup \mathscr{F}, p, q, \mathscr{F})$ by letting $(a \rho) \Phi$ be the restriction of $a$ to $\cup \mathscr{F}$. If $a \rho=b \rho$ and $x \in \cup \mathscr{F}$ choose $d \in B B L(\cup \mathscr{F}, p, q, \mathscr{F})$ and $y \in \cup \mathscr{F}$ so that $(y) d=x$. Hence $(x) a=(y) d a=$ $(y) d b=(x) b$. Thus $\Phi$ is a function. That $\Phi$ is a one-to-one homomorphism follows from the fact that if $b \in B$ then $X b \subset \cup \mathscr{F}$. That $\Phi$ is onto follows from property (B2) for $\mathscr{F}$.

For the remainder of this section we assume that $|X|=p$ is infinite, $q$ is an infinite cardinal number, $q \leqq p, \mathscr{F}$ is an effective bounding collection for $X$, and $B=B B L(X, p, q, \mathscr{F})$.

If $a$ and $b$ are transformations of $X$ then the difference set of $a$ and $b$, noted $D(a, b)$, is the set $\{x \in X: x a \neq x b\}$. The following lemma is routine to verify but is frequently used hereafter.

LEMMA 2.2. If $a, b$, and $c$ are transformations of $X$, then

(i) $D(a, c) \subset D(a, b) \cup D(b, c)$;

(ii) $D(c a, c b)=(D(a, b)) c^{-1}$; and

(iii) if $c$ is one-to-one, then $D(a c, b c)=D(a, b)$.

If $l$ is a cardinal number, we note by $l^{+}$the smallest cardinal number larger than $l$. If $r$ is a cardinal number and $\aleph_{0} \leqq r \leqq p^{+}$, define the $r$-difference relation on $B$ by $\delta_{r}=\{(a, b) \in B \times B:|D(a, b)|<r\}$. Define the $r(\mathscr{F})$ difference relation on $B$ by

$$
\delta(\mathscr{F})_{r}=\{(a, b) \in B \times B:|D(a, b) \cap F|<r \text { for each } F \in \mathscr{F}\}
$$

We argue now that this relation is independent of the choice of a bounding 
collection yielding the same $B$. We recall that $B B L(X, p, q, \mathscr{F})=$ $B B L(X, p, q, \mathscr{G})$ is equivalent to $\mathscr{F}$ and $\mathscr{G}$ being interlaced, i.e., if $F \in \mathscr{F}$ there is $G \in \mathscr{G}$ so that $F \subset G$ and vice versa. Thus if so and $(a, b) \in \delta(\mathscr{F})_{\text {r }}$, i.e., $|D(a, b) \cap F|<r$ for each $F \in \mathscr{F}$, then $|D(a, b) \cap G|<r$ for each $G \in \mathscr{G}$ and $(a, b) \in \delta(\mathscr{G})_{r}$. Thus $\delta(\mathscr{F})_{r} \subset \delta(\mathscr{G})_{r}$ and vice versa.

We single out the following statement for emphasis.

Proposition 2.3. For any infinite cardinal number $r, \delta_{r} \subset \delta(\mathscr{F})_{\text {. }}$.

Proposition 2.4. The relations $\delta$, and $\delta(\mathscr{F})$, are congruences on $B$.

Proof. It follows from Lemma 2.2 that $\delta$, is a congruence and $\delta(\mathscr{F})_{r}$ is a right compatible equivalence relation. To see that $\delta(\mathscr{F})$, is left compatible let $(a, b) \in \delta(\mathscr{F})$, and $s \in B$. There is $F \in \mathscr{F}$ so that $X s \subset F$, and, by Lemma 2.2, $D(s a, s b)=D(a, b) s^{-1}$. Therefore

$$
D(a, b) s^{-1}=D(a, b) s^{-1} \cap F s^{-1}=(D(a, b) \cap F) s^{-1}
$$

and the latter set has cardinality less than $r$ since $s$ is one-to-one and $(a, b) \in$ $\delta(\mathscr{F})_{r}$. Consequently $(s a, s b) \in \delta_{r} \subset \delta(\mathscr{F})_{r}$.

Šutov (1961) has shown that in the case where $p=q$ the only proper congruences on $B L(X, p, p)$ are the $r$-difference congruences; in fact, the following proofs are similar to proofs of some of Šutov's results. From Lindsey and Madison (1976) the only non-trivial non-group congruences on $B L(X, p, q)$ are the $r$-difference congruences. As we see in Section 3 this is not the case with $B B L(X, p, q, \mathscr{F})$.

Proposition 2.5. If $|\mathscr{F}| \leqq r$ then $\delta(\mathscr{F}), \subset \delta_{r}{ }^{+}$.

Proof. (We first remark that the $c(\mathscr{F})$ defined in Section 1 can be used instead of $|\mathscr{F}|$.) If $(a, b) \in \delta(\mathscr{F})_{r}$ then $|D(a, b)|=\left|\bigcup_{F \in \mathbb{F}}(D(a, b) \cap F)\right| \leqq$ $\boldsymbol{r} \cdot \boldsymbol{r} \equiv \boldsymbol{r}<\boldsymbol{r}^{+}$.

We note that $\delta_{r^{+}}$is never a subset of $\delta(\mathscr{F})_{m}$, i.e., if $\delta(\mathscr{F})_{r} \not \subset \delta_{r^{+}}$then these congruences do not compare.

Proofs of the next two lemmas are given in Lindsey and Madison (1976).

LEMMA 2.6. If $a, b \in B$ and $D$ is an infinite subset of $D(a, b)$, then there is a subset $Y$ of $D$ with $|Y|=|D|$ so that $Y a \cap Y b=\varnothing$.

LEMMA 2.7. If $\rho$ is a congruence on a semigroup $S,(a, b) \in \rho$, and for some $s, t, u \in S, a t=a u$ and $b s=b u$, then $(a s, b t) \in \rho$.

LEMMA 2.8. If $\sigma$ is a congruence on $B, F \in \mathscr{F}$, and there is $(a, b) \in \sigma$ with $|D(a, b) \cap F|>q$, then there is $\left(a^{\prime}, b^{\prime}\right) \in \sigma$ with $\left|D\left(a^{\prime}, b^{\prime}\right) \cap F\right|=q$.

Proof. By Lemma 2.6 choose $Y \subset D(a, b) \cap F$ so that $|Y|=q$ and 
$Y a \cap Y b=\varnothing$. Note that $Y \cap Y b a^{-1}=\varnothing$. Define $c \in B$ so that $(y) c=y$ for each $y \in Y$ and $Y b a^{-1} \cap X c=\varnothing$. This is clearly possible since $\mid F \backslash(Y \cup$ $\left.Y b a^{-1}\right) \mid=p$ and the hypotheses imply that $p>q$. Note that $X c a \cap Y c b=\varnothing$ since if not there is $x \in X$ and $y \in Y$ so that $(x) c a=(y) c b=(y) b$ and thus $(x) c=(y) b a^{-1}$ contradicting $Y b a^{-1} \cap X c=\varnothing$. Define $t \in B$ so that $(x) t=x$ if $x \in(X c a \cup X c b) \backslash Y c b$ and $(x) t \neq x$ if $x \in Y c b$. Since there are $F_{1}, F_{2} \in \mathscr{F}$ so that $X c a \cup X c b \subset F_{1} \subset F_{2}$ and $\left|F_{2} \backslash F_{1}\right|=q, t$ can map $X \backslash(X c a \cup X c b) \cup Y c b$ into $F_{2} \backslash F_{1}$. Now $(c a t, c b t)=(c a, c b t) \in \sigma$ and $(c a, c b) \in \sigma$ imply that $(c b, c b t) \in \sigma$. Now if $x \in Y,(x) c b t \neq(x) c b$. If $x \in X \backslash Y$ then $(x) c b t=(x) c b$. Consequently $D(c b, c b t)=Y$. Since $Y \subset F, D(c b, c b t) \cap F=Y$.

LEMMA 2.9. If $\sigma$ is a congruence on $B, r$ is a cardinal number, $\boldsymbol{N}_{0} \leqq r \leqq q$, and there is $F \in \mathscr{F}$ and $(a, b) \in \sigma$ with $|D(a, b) \cap F|=r$ then $\delta_{r}+\subset \sigma$.

Proof. By Lemma 2.6 there is a subset $Y \subset D(a, b) \cap F$ so that $|Y|=$ $|D(a, b) \cap F|=r$ and $Y a \cap Y b=\varnothing$. Let $(c, d) \in \delta_{r^{+}}$, and denote $D(c, d)$ by $D$ for convenience. We show that $(c, d) \in \sigma$. If $r=p$, choose $s \in B$ so that $X s \subset Y$. If $r \neq p$, choose $s \in B$ so that $D s \subset Y$ and $(X \backslash D) s \subset[X \backslash D(a, b)] \cap F$. This is possible since $|D| \leqq|Y|$ and $|(X \backslash D(a, b)) \cap F|=p$. Now $(s a, s b) \in \sigma$ and since $D s a \subset Y a$ and $D s b \subset Y b$, we have $D s a \cap D s b=\varnothing$. Let $t_{1} \in B$ be defined so that on $X s a, t_{1}=(s a)^{-1} c$, i.e., sat $t_{1}=c$. There are $F, F^{\prime} \in \mathscr{F}$ so that $X c \subset F \subset F^{\prime}$ and $\left|F^{\prime} \backslash F\right|=q$. Let $t_{1}$ map $X \backslash X s a$ into $F^{\prime} \backslash F$. Define $t_{2} \in B$ so that $t_{2}=(s b)^{-1} d$ on $X s b$. There are $F^{\prime \prime}, F^{\prime \prime \prime} \in \mathscr{F}$ with $X d \subset F^{\prime \prime}, F^{\prime} \cup F^{\prime \prime} \subset F^{\prime \prime}$, and $\left|F^{\prime \prime \prime} \backslash F^{\prime} \cup F^{\prime \prime}\right|=q$. Let $t_{2}$ map $X \backslash X s b$ into $F^{\prime \prime \prime} \backslash\left(F^{\prime} \cup F^{\prime \prime}\right)$. Since $D s b t_{1} \cap$ $D_{s a t_{2}}=\varnothing$ and $D(s a, s b)=D \equiv D(c, d)$ we note that if $z \in X s a \cap X s b$ then $z=x s a=x s b$ for $x \in X \backslash D$. Thus $(x) t_{1}=(x) c=(x) d=(x) t_{2}$. Consequently we can define $t \in B$ to agree with $t_{1}$ on $X s b$ and to agree with $t_{2}$ on $X s a$, i.e., $s b t=s b t_{1}$ and $s a t=s a t_{2}$. Since $\left|X \backslash\left(X t_{1} \cup X t_{2}\right)\right|=q$ there is sufficient room to complete the definition of $t$. Finally $\left(s b t_{1}, s a t_{2}\right)=(s b t, s a t) \in \sigma,\left(s b t_{1}, s a t_{1}\right) \in \sigma$, and $\left(s b t_{2}, s a t_{2}\right) \in \sigma$. Thus $(c, d)=\left(s a t_{1}, s b t_{2}\right) \in \sigma$.

COROLlaRY 2.10. If $p=q$ then $\delta(\mathscr{F})_{p}$ is the unique maximum congruence on $B$.

Proof. Apply Lemma 2.9 with $r=p=q$.

Whether or not $\delta(\mathscr{F})_{p}$ is a maximal (or the unique maximum) congruence on $B$ when $p \neq q$ is unknown to the authors. In the case that $p \neq q$ the congruence $\delta(\mathscr{F})_{p}$ induces a congruence on the group $B / \delta_{q^{+}}$. (See 2.14 below.) The authors expect to investigate this group and its congruences in future work.

CoROllary 2.11. Let $\kappa_{0} \leqq r \leqq q$. If $\sigma$ is a congruence on $B$, either $\sigma \subset \delta(\mathscr{F})$, or $\delta_{r}+\subset \sigma$. 
Proof. If $\sigma \not \subset \delta(\mathscr{F})_{r}$ there is $(a, b) \in \sigma \backslash \delta(\mathscr{F})_{r}$, i.e., for some $F \in \mathscr{F}$, $|D(a, b) \cap F| \geqq r$. By Lemma 2.8 there is $\left(a^{\prime}, b^{\prime}\right) \in \sigma$ so that $\mid D\left(a^{\prime}, b^{\prime}\right) \cap$ $F \mid=r$ and by Lemma 2.9, $\delta_{r^{+}} \subset \sigma$.

Corollary 2.12. If $\aleph_{0} \leqq r \leqq q$ then $\delta(\mathscr{F})_{r} \wedge \delta_{r^{+}}$is the unique maximal congruence on $B$ below $\delta_{r^{+}}$.

Proof. Apply Corollary 2.11.

LEMMA 2.13. The quotient semigroup $B / \delta(\mathscr{F})_{q}$ is idempotent-free.

Proof. For any $a \in B$ we show $\left(a, a^{2}\right) \notin \delta(\mathscr{F})_{q}$. There are $F, F^{\prime} \in \mathscr{F}$ so that $X a \subset F \subset F^{\prime}$ and $\left|F^{\prime} \backslash F\right|=q$. Then $F^{\prime} \mid F \subset X \backslash X a \subset\{X: x a \neq x\}=$ $D\left(a, a^{2}\right)$. Thus $\left|D\left(a, a^{2}\right) \cap F^{\prime}\right| \geqq q$ or $\left(a, a^{2}\right) \notin \delta(\mathscr{F})_{q}$.

Proposition 2.14. The congruence $\delta_{q^{+}}$is the unique minimal group congruence on $B$.

Proof. If $p=q$ then $\delta_{q^{+}}=B \times B$ and there is a unique maximal congruence, namely $\delta(\mathscr{F})_{q}$ by 2.10 , below $\delta_{q^{+}}$and by $2.13, B / \delta(\mathscr{F})_{q}$ is not a group. If $q<p$ and $a \in B$ then $\left(a, a^{2}\right) \in \delta_{q^{+}}$if and only if $a$ is the identity on a set $A$, $|A|=p$, and $|X| A \mid=q$. To see this note that $D\left(a, a^{2}\right)$ is the set $X \backslash\{x: x a=$ $x$. Clearly there is such an $a$ in $B$. Further any two such elements of $B$, say $a$ and $a^{\prime}$, are $\delta_{q}+$ related. Consequently $B / \delta_{q}+$ has a unique idempotent and this along with right simplicity yields a group. To see that $\delta_{q}+$ is minimal let $\sigma$ be a group congruence on $B$. By Corollary 2.11 either $\sigma \subset \delta(\mathscr{F})_{q}$ or $\delta_{q}+\sigma \sigma$. Since by Lemma $2.13 B / \delta(\mathscr{F})_{q}$ is idempotent-free it cannot be that $\sigma \subset \delta(\mathscr{F})_{q}$. Thus $\delta_{q}+\subset \sigma$.

Proposition 2.15. The $\boldsymbol{N}_{0}$-difference set congruence $\delta_{\boldsymbol{n}_{0}}$ is the unique minimal congruence on $B$.

Proof. The proof that $\delta_{\boldsymbol{w}_{0}}$ is minimal is the same as the proof that $\delta_{\boldsymbol{m}_{0}}$ is minimal on $B L(X, p, q)$, Lindsey and Madison (1976). To see that $\delta_{m_{0}}$ is unique minimal let $\sigma$ be a non-trivial congruence on $B$. By Lemma 2.11 either $\sigma \subset \delta(\mathscr{F})_{\boldsymbol{\kappa}_{0}}$ or $\delta_{\boldsymbol{\mu}_{0}} \subset \sigma$. If $\sigma \subset \delta(\mathscr{F})_{\boldsymbol{\kappa}_{0}}$ let $(a, b) \in \sigma$ with $a \neq b$. There is $F \in \mathscr{F}$ so that $D(a, b) \cap F \neq \varnothing$. Choose $s \in B$ so that $X s=F$. Then $D(s a, s b) \neq \varnothing$ and is finite since $(a, b) \in \delta(\mathscr{F})_{\boldsymbol{\alpha}_{0}}$. Consequently $\sigma \cap \delta_{\boldsymbol{\alpha}_{0}} \neq \Delta$ and hence since $\delta_{\aleph_{0}}$ is minimal $\sigma \cap \delta_{\aleph_{0}}=\delta_{\aleph_{0}}$, i.e., $\delta_{\aleph_{0}} \subset \sigma$.

\section{Other congruences on $B B L(X, p, q, \mathscr{F})$}

According to Corollary 2.12 , if $r$ is a cardinal number with $\aleph_{0} \leqq r \leqq q$, then there are no congruences between $\delta(\mathscr{F})_{r} \cap \delta_{r^{+}}$and $\delta_{r^{+}}$. We see here that, 
in general, there are many congruences between $\delta_{r}$ and $\delta(\mathscr{F})_{r}$. We will construct two classes of congruences that are in this interval and discuss their properties. Again let $B=B B L(X, p, q, \mathscr{F})$.

If $C$ and $D$ are subsets of $X$ we say that $C$ is $r$-almost contained in $D$, written $C C_{r} D$ if $|C \backslash D|<r$. The sets $C$ and $D$ are said to be $r$-almost equal, written $C={ }_{r} D$, if $C \subset_{r} D$ and $D \subset_{r} C$.

If $r$ is a cardinal number and $A \subset X$, then we will note by $\sigma_{r}(A)$ the set $\left\{(a, b) \in B \times B: D(a, b) \subset_{r} A\right\}$. The set $A$ will be said to be $r$-scattered in $\mathscr{F}$ if for each $F \in \mathscr{F},|F \cap A|<r$. We will note by $S_{c}(\mathscr{F})$ the set of subsets of $X$ that are $r$-scattered in $f$.

Proposition 3.1. If $A \in S c_{r}(\mathscr{F})$ then $\sigma_{r}(A)$ is a congruence on $B$ and $\delta_{r} \subset$ $\sigma_{r}(A) \subset \delta(\mathscr{F})_{r}$

Proof. First $\delta_{r} \subset \sigma_{r}(A) \subset \delta(\mathscr{F})$, is clear. Also it is easy to see that $\sigma_{r}(A)$ is a right compatible equivalence relation. If $b \in B$ and $(c, d) \in \sigma_{r}(A)$ then since $A \in S c_{r}(\mathscr{F})$ and $b$ is bounded it follows that $(b c, b d) \in \delta_{r}$.

We note that if $|A|<r$ then $\sigma_{r}(A)=\delta_{r}$.

Proposition 3.2. If $A, A^{\prime} \in S c_{r}(\mathscr{F})$ then in the lattice of congruences on $B$ we have $\sigma_{r}(A) \wedge \sigma_{r}\left(A^{\prime}\right)=\sigma_{r}\left(A \cap A^{\prime}\right)$.

PRoof. Now $\sigma_{r}(A) \wedge \sigma_{r}\left(A^{\prime}\right)=\sigma_{r}(A) \cap \sigma_{r}\left(A^{\prime}\right)$ and the result follows set theoretically. $\square$

Lemma 3.3. If $a, b \in B, \quad A \subset X, \quad A a \cap A b=\varnothing, \quad D(a, b)=A, \quad$ and $c, d \in B$ with $D(c, d) \subset A$, then $(c, d) \in\langle(a, b)\rangle$, the smallest congruence containing the pair $(a, b)$.

Proof. First we note that $|A| \leqq q$ since $D(a, b)=A$ and $A a \cap A b=\varnothing$. Thus there are functions $s, t \in B$ so that $a s=c, b t=d$, and $A b s \cap A a t=\varnothing$. Further, there is a function $u \in B$ which agrees with $s$ on $X b$, agrees with $t$ on $X a$, and assumes the common value of $s$ and $t$ on $X \backslash(A a \cup A b)$. Hence $b s=$ $b u$ and $a t=a u$. By Lemma $2.7(c, d)=(a s, b t) \in\langle(a, b)\rangle$.

Define $S c_{r}^{*}(\mathscr{F})=\left\{A \in S c_{r}(\mathscr{F}):|A| \leqq q\right.$ and for each cardinal number $\hat{r}<r$ there is $F \in \mathscr{F}$ so that $|F \cap A| \geqq \hat{r}\}$.

We will call a congruence $\sigma$ on a semigroup $S$ monogenic if there is a pair $(a, b) \in S \times S$ so that $\sigma=\langle(a, b)\rangle$.

Proposition 3.4. If $\boldsymbol{\aleph}_{o} \leqq r \leqq q$ and $A \in S_{c}^{*}(\mathscr{F})$, then $\sigma_{r}(A)$ is a monogenic congruence on $B$.

Proof. Let $a, b \in B$ so that $D(a, b)=A$ and $A a \cap A b=\varnothing$. This is possible since $|A| \leqq q$. Let $(c, d) \in \sigma_{r}(A)$, i.e., $D(c, d) C_{r} A$. Let $c^{\prime}, d^{\prime} \in B$ where $c^{\prime}=c$ except on $D(c, d) \backslash A, d^{\prime}=d$ except on $D(c, d) \backslash A$, and $c^{\prime}=d^{\prime}$ on 
$D(c, d) \backslash A$. Then $D\left(c^{\prime}, d^{\prime}\right) \subset A$ and by Lemma $3.3,\left(c^{\prime}, d^{\prime}\right) \in\langle(a, b)\rangle$. Further $\left(c, c^{\prime}\right)$ and $\left(d, d^{\prime}\right)$ are in $\delta_{r}$. We now claim that $\delta_{r} \subset\langle(a, b)\rangle$. To see this we note that $A \in S c_{r}^{*}(\mathscr{F})$ and Lemma 2.9 yield the following: If $\hat{r}$ is a cardinal number and $\hat{r}<r$ there is cardinal number $\bar{r} \geqq \hat{r}$ so that $\delta_{\bar{r}^{+}} \subset\langle(a, b)\rangle$. Now $(a, b) \in \delta_{r}$ implies $|D(a, b)|=\hat{r}<r$ and thus $(a, b) \in S_{r^{+}}$. Further if $\hat{r}<r$ and $(a, b) \in S_{r^{+}}$ then $|D(a, b)|<\hat{r}^{+} \leqq r$. Thus $(a, b) \in \delta_{r}$. It follows that $\delta_{r}=\bigcup_{r<r} \delta_{f^{+}}$and that $\delta, \subset\langle(a, b)\rangle$. Consequently $\left(c, c^{\prime}\right),\left(c^{\prime}, d^{\prime}\right)$, and $\left(d^{\prime}, d\right)$ are all in $\langle(a, b)\rangle$. By transitivity, $(c, d) \in\langle(a, b)\rangle$ and $\sigma_{r}(A)=\langle(a, b)\rangle$.

Proposition 3.5. In the lattice of congruences on $B$, if $A$ and $A^{\prime}$ are in $S c_{r}^{*}(\mathscr{F})$ then $\sigma_{r}(A) \vee \sigma_{r}\left(A^{\prime}\right)=\sigma_{r}\left(A \cup A^{\prime}\right)$.

Proof. If $A$ and $A^{\prime}$ are in $S c_{r}^{*}(\mathscr{F})$ then so is $A \cup A^{\prime}$. Hence $\sigma_{r}\left(A \cup A^{\prime}\right)$ is a congruence on $B$ and $\sigma_{r}(A) \vee \sigma_{r}\left(A^{\prime}\right) \subset \sigma_{r}\left(A \cup A^{\prime}\right)$. Now let $(a, b) \in$ $\sigma_{r}\left(A \cup A^{\prime}\right)$ so that $D(a, b)=A \cup A^{\prime}$ and $\left(A \cup A^{\prime}\right) a \cap\left(A \cup A^{\prime}\right) b=\varnothing$. Define $s \in B$ by $(x) s=(x) a$ if $x \in A,(x) s=(x) b$ is $x \in A^{\prime} \backslash A$, and $(x) s=$ $(x) a=(x) b$ if $x \in X \backslash\left(A \cup A^{\prime}\right)$. Then $D(a, s) \subset A^{\prime}$ and $D(s, b)=A$. Thus $(a, s) \in \sigma_{r}\left(A^{\prime}\right)$ and $(s, b) \in \sigma_{r}(A)$, and hence $(a, b) \in \sigma_{r}(A) \vee \sigma_{r}\left(A^{\prime}\right)$. Thus $\langle(a, b)\rangle \subset \sigma_{r}(A) \vee \sigma_{r}\left(A^{\prime}\right)$ and by Proposition 3.4 and its proof, $\sigma_{r}\left(A \cup A^{\prime}\right)=$ $\langle(a, b)\rangle$.

We observe that in many special cases the previous result yields a distributive sublattice of the lattice of congruences on $B$. The simplest case is where $p=q=r=\aleph_{0}$. Other such cases result when the cardinality of $\mathscr{F}$ is "small".

We now define another collection of congruences on $B$. If $\mu \subset 2^{x}$ and $r$ is an infinite ca, dinal number we set $\rho_{r}(\mathcal{M})=\left\{(a, b) \in \delta(\mathscr{F})_{r}: M a={ }_{r} M b\right.$ for all $M \in \mathcal{M}\}$. Now if $\mathscr{A}=\left\{\mathscr{M}^{\prime} \subset 2^{x}: \rho_{r}\left(\mathcal{M}^{\prime}\right)=\rho_{r}(\mathcal{M})\right\}$ then $\rho_{r}(\mathcal{M})=\rho(\cup \mathscr{A})$ and $\cup \mathscr{A}$ is the unique maximal collection $\mathscr{C}$ so that $\rho_{r}(\mathscr{C})=\rho_{r}(\mathcal{M})$. We shall frequently assume that we are representing $\rho_{r}(\mathcal{M})$ by using this maximal collection as $\mathcal{M}$.

PROPOSITION 3.6. If $\mathcal{M} \subset 2^{x}$ and $r$ is an infinite cardinal number, then $\rho_{r}(\mathcal{M})$ is a congruence on $B$ and $\delta, \subset \rho_{r}(\mathcal{M}) \subset \delta(\mathscr{F})$.

PROOF. That $\rho_{r}(\mathcal{M})$ is a right compatible equivalence relation is clear. If $M \in \mathcal{M}, b \in B$, and $(c, d) \in \rho_{r}(\mathcal{M}) \subset \delta(\mathscr{F})_{r}$, then there is $F \in \mathscr{F}$ with $M b \subset F$. Further $|D(c, d) \cap F|<r$. Consequently $M b c=, M b d$, and hence $(b c, b d) \in$ $\rho_{r}(\mathcal{M})$. The containments are obvious.

PROPOSITION 3.7. Let $\rho_{r}(\mathcal{M})$ and $\rho_{r}\left(\mathcal{M}^{\prime}\right)$ be congruences of the type above with $\mathcal{M}$ and $\mathcal{M}^{\prime}$ maximal collections. Then $\rho_{r}(\mathcal{M}) \subset \rho_{r}\left(\mathcal{M}^{\prime}\right)$ if and only if $\mathcal{M}^{\prime} \subset \mathcal{M}$. 
Proof. If $\mathcal{M}^{\prime} \subset \mathcal{M}$ then clearly $\rho_{r}(\mathcal{M}) \subset \rho_{r}\left(\mathscr{M}^{\prime}\right)$. If $M^{\prime} \in \mathcal{M}^{\prime} \backslash \mathcal{M}$, since $\mathcal{M}$ is maximal, there is a pair $(a, b) \in B \times B$ so that $M a=, M b$ for each $M \in \mathcal{M}$ but $M^{\prime} a \neq{ }_{r} M^{\prime} b$. Consequently if $\mathcal{M}^{\prime} \not \subset \mathcal{M}$ then $\rho_{r}(\mathcal{M}) \not \subset \rho_{r}\left(\mathcal{M}^{\prime}\right)$.

Proposition 3.8. The collection $\left\{\rho_{r}(\mathcal{M}): \mathcal{M} \subset 2^{x}\right\}$ is a distributive lattice of congruences on $B$.

Proof. From 3.7 it is easy to see that $\rho_{r}(\mathcal{M}) \vee \rho_{r}\left(\mathcal{M}^{\prime}\right)=\rho_{r}\left(\mathcal{M} \cap \mathcal{M}^{\prime}\right)$ and $\rho_{r}(\mathcal{M}) \wedge \rho_{r}\left(\mathcal{M}^{\prime}\right)=\rho_{r}\left(\mathcal{M} \cup \mathcal{M}^{\prime}\right)$. (We do not claim that this supremum is the supremum in the lattice of all congruences on $B$.) The distributivity follows.

The next two results compare the $\rho_{r}(\mathcal{M})$ 's and the $\rho_{r}(A)$ 's.

Proposition 3.9. Let $\mu \subset 2^{x}$ and assume it is the maximal collection for the congruence $\rho_{r}(\mathcal{M})$. Let $A \in S c_{r}(\mathscr{F})$. Then $\rho_{r}(\mathcal{M}) \subset \rho_{r}(A)$ if and only if $2^{X \backslash A} \subset \mathcal{M}$.

Proof. Suppose $\rho_{r}(\mathcal{M}) \subset \sigma_{r}(A)$. Let $(a, b) \in \rho_{r}(\mathcal{M})$ and $J \in 2^{X \backslash A}$. Since $D(a, b) \subset, A$ it follows that $J a=, J b$ and hence $J \in \mathcal{M}$ since $\mathcal{M}$ is maximal. Conversely, suppose $2^{X \backslash A} \subset \mathcal{M}$. Let $(a, b) \in \rho_{r}(\mathcal{M})$. If there is a set $J \subset X \backslash A$, $|J| \geqq r$, and $J \subset D(a, b)$, then by Lemma 2.6 there is a set $Y \subset J,|Y|=r$ so that $Y a \cap Y b=\varnothing$. Thus $Y \notin \mathcal{M}$. Consequently $D(a, b) \subset, A$.

Proposition 3.10. Let $\mu \subset 2^{x}, \quad r \leqq q$, and $A \in S c_{r}(\mathscr{F})$. Then $\sigma_{r}(A) \subset \rho_{r}(\mathcal{M})$ if and only if for each $M \in \mathcal{M}, M \subset_{r} X \backslash A$.

Proof. Suppose $m \in \mathcal{M}$ and $M \not \subset, X \backslash A$, i.e., $|M \cap A| \geqq r$. Let $Y \subset M \cap A$ with $|Y|=r$. Since $r \leqq q$ there are $a, b \in B$ so that $Y a \cap Y b=$ $\varnothing$ and $a$ and $b$ agree otherwise. Then $(a, b) \in \sigma_{r}(A)$ and $(a, b) \notin \rho_{r}(\mathcal{M})$. Thus $\sigma_{r}(A) \not \subset \rho_{r}(\mathcal{M})$. Conversely, if $(a, b) \in \sigma_{r}(A)$ then $D(a, b) \subset_{r} A$. Thus if $M \in$ $\mathcal{M}$ we have $M \subset, X \backslash A \subset, X \backslash D(a, b)$. Therefore $M a={ }_{r} M b$ and $(a, b) \in$ $\rho_{r}(\mathcal{M})$.

Although we do not know if all congruences on $B$ can be described in terms of those given here, one can routinely conclude some properties of quotients of $B$. If $\rho$ is any congruence on $B$ then $B / \rho$ is right simple and right cancellative, and any two elements of $B / \rho$ have a common right identity. Further, either $B / \rho$ is a group or $E(B / \rho)=\varnothing$. If, in addition, $\delta_{r} \leqq \rho<\delta(\mathscr{F})$ r then $B / \rho$ is not left reductive.

We conclude with a simple example of a bounded Baer-Levi semigroup $B(X, p, q, \mathscr{F})$ where the four types of congruences (for a given $r$ ) are different.

Let $X=\{1,2,3, \cdots\}, p=q=r=\aleph_{0}=|X|$, and let $\left\{A_{i}: i=1,2,3, \cdots\right\}$ be a collection of pairwise disjoint subsets of $X$ with $\left|A_{i}\right|=\boldsymbol{N}_{0}$ for each $i$ and $X=\bigcup_{i=1}^{\infty} A_{i}$. Let $F_{n}=\bigcup_{i=1}^{n} A_{i}$ and $\mathscr{F}=\left\{F_{n}: n=1,2,3, \cdots\right\}$. It is clear that $\delta_{\aleph_{0}}$ is properly contained in $\delta(\mathscr{F})_{N_{0}}$. Choose $A \subset X$ with $0<\left|A \cap A_{i}\right|<\aleph_{0}$ for 
each $i$. Then $\left|A \cap F_{n}\right|<\boldsymbol{N}_{0}$ for each $n$ and $|A|=\boldsymbol{N}_{0}$. Consequently $A \in S c_{\aleph_{0}}(\mathscr{F})$ and $\sigma_{\mathcal{N}_{0}}(A)$ is a congruence on $B=B\left(X, \boldsymbol{N}_{0}, \boldsymbol{N}_{0}, \mathscr{F}\right)$. It is easy to see that $\sigma_{\boldsymbol{N}_{0}}(A)$ is properly between $\delta_{\boldsymbol{N}_{0}}$ and $\delta(\mathscr{F})_{\boldsymbol{\mu}_{0}}$. Now choose $\mathcal{M}=\{A\}$. From Propositions 3.9 and 3.10 it follows that $\rho_{\mathcal{M}_{0}}(\mathcal{M})$ and $\sigma_{\mathcal{M}_{0}}(A)$ do not comjare. From Proposition 3.6 we know that $\delta_{\boldsymbol{N}_{0}} \subset \rho_{\boldsymbol{\kappa}_{0}}(\mathcal{M}) \subset \delta(\mathscr{F})_{\boldsymbol{M}_{0}}$. Choose $a, b \in$ $B$ so that $D(a, b)=A$ and $A a \cap A b=\varnothing$. Then $(a, b) \in \delta(\mathscr{F})_{\kappa_{0}}$ and $(a, b) \notin \rho_{\aleph_{0}}(\mathcal{M})$. Clearly there are pairs $(c, d) \in \rho_{\boldsymbol{N}_{0}}(\mathcal{M})$, i.e., $A c$ and $A d$ differ sy finitely many points, and $|D(c, d)|=\mathcal{N}_{0}$. Consequently $\rho_{\mathcal{N}_{0}}(\mathcal{M}) \not \subset \delta_{\boldsymbol{N}_{0}}$. Hence $\delta_{\boldsymbol{\kappa}_{0}}, \sigma_{\boldsymbol{N}_{0}}(A), \rho_{\boldsymbol{\kappa}_{0}}(\mathcal{M})$, and $\delta(\mathscr{F})_{\boldsymbol{N}_{0}}$ are distinct congruences in $B$.

\section{References}

२. Baer and F. Levi (1932), 'Vollständige irreduzibele Systeme von Gruppenaxiomen', Heidelberger Akad. Wiss. Abh. 2, 1-12.

A. H. Clifford and G. B. Preston (1961), The Algebraic Theory of Semigroups, vol. I (Amer. Math. Soc., Providence).

A. H. Clifford and G. B. Preston (1967), The Algebraic Theory of Semigroups, vol. II (Amer. Math. Soc., Providence).

J. Lindsey (1974), 'Relations invariant under semigroup actions', Semigroup Forum 8, 298-311.

). Lindsey (1975), 'Relations invariant under semigroup actions and bounded Baer-Levi semigroups', Dissertation, Louisiana State University.

). Lindsey and B. Madison (1976), 'The lattice of congruences on a Baer-Levi semigroup', Semigroup Forum 12, 63-70.

3. W. Mielke (1972), 'Regular congruences on Croisot-Teissier and Baer-Levi semigroups', $J$. Math. Soc. Japan 24, 539-551.

3. W. Mielke (1973), 'Regular congruences on a simple semigroup with a minimal right ideal', Publ. Math. Debrecen 20, 79-84.

3. W. Mielke (1975), 'Completely simple congruences on Croisot-Teissier semigroups', Semigroup Forum 9, 283-293.

ミ. G. Šutov (1961), 'Semigroups of one-to-one transformations', Dokl. Akad. Nauk. SSSR. 140, 1026-1028.

-ouisiana State University, 3aton Rouge, Louisiana 70803

J.S.A.

ind

Jniversity of South Alabama,

Mobile, Alabama, 36688

J.S.A. 